\title{
Single Mode Photonic Crystal Vertical Cavity Surface Emitting Lasers
}

\author{
Kent D. Choquette, ${ }^{1}$ Dominic F. Siriani, ${ }^{1}$ Ansas M. Kasten, ${ }^{2}$ Meng Peun Tan, ${ }^{1}$ Joshua D. \\ Sulkin, ${ }^{1}$ Paul O. Leisher, ${ }^{3}$ James J. Raftery Jr., ${ }^{4}$ and Aaron J. Danner ${ }^{5}$ \\ ${ }^{1}$ Department of Electrical and Computer Engineering, University of Illinois, Urbana, IL, 61801, USA \\ ${ }^{2}$ Micro and Nano Structures Technologies, GE Global Research, Niskayuna NY 12309, USA \\ ${ }^{3}$ Department of Physics and Optical Engineering, Rose-Hulman Institute of Technology, Terre Haute, IN 47803, USA \\ ${ }^{4}$ Department of Electrical Engineering, United States Military Academy, West Point, NY 10996, USA \\ ${ }^{5}$ Department of Electrical and Computer Engineering, National University of Singapore, Singapore 117576
}

Correspondence should be addressed to Kent D. Choquette, choquett@illinois.edu

Received 27 July 2011; Accepted 5 October 2011

Academic Editor: Krassimir Panajotov

Copyright ( $) 2012$ Kent D. Choquette et al. This is an open access article distributed under the Creative Commons Attribution License, which permits unrestricted use, distribution, and reproduction in any medium, provided the original work is properly cited.

We review the design, fabrication, and performance of photonic crystal vertical cavity surface emitting lasers (VCSELs). Using a periodic pattern of etched holes in the top facet of the VCSEL, the optical cavity can be designed to support the fundamental mode only. The electrical confinement is independently defined by proton implantation or oxide confinement. By control of the refractive index and loss created by the photonic crystal, operation in the Gaussian mode can be insured, independent of the lasing wavelength.

\section{Introduction}

Vertical cavity surface emitting lasers (VCSELs) have emerged as the commercial laser source of choice for short distance digital fiber optical interconnects and sensing applications. The principle advantage that VCSELs have in many of these applications are their low operating power requirements (only a few $\mathrm{mW}$ ) as well as their low cost and large volume manufacturing. Compared to edge emitting semiconductor lasers, VCSELs also possess the benefits of a circular output beam, on wafer testing, and the ability to form 2-dimensional arrays. The most common emission wavelength is $850 \mathrm{~nm}$, although wavelengths from 640 to $1300 \mathrm{~nm}$ have been demonstrated for VCSELs monolithically grown on GaAs substrates.

Unlike an edge emitting laser, VCSELs have a single longitudinal mode but tend to operate in multiple transverse optical modes. This arises because the optical cavity of the VCSEL is short in the direction of light propagation (typically the cavity is 1 wavelength long, or approx. $265 \mathrm{~nm}$ for $850 \mathrm{~nm}$ emission), but the transverse cavity width defined by a selectively oxidized or ion implanted aperture [1] is much greater (typically a few to tens of microns in diameter). Edge emitting semiconductor lasers have much longer cavities (several hundreds of microns in length) supporting numerous longitudinal modes, but with a cavity cross-section that supports a few or a single transverse/lateral mode. The number of laser emission modes will influence the spectral width of the laser emission, while the near field and far field beam profile is determined by the transverse mode profiles. For a typical multimode $850 \mathrm{~nm}$ VCSEL, the emission bandwidth can be roughly $3 \mathrm{~nm}$, while the far field is often a ring shape, due to the higher order mode operation. The multimode VCSEL bandwidth can be a limiting factor for high speed digital modulation through optical fiber due to spectral dispersion, while the smallest focused spot size will come from operation only in the fundamental Gaussian mode.

Many approaches to achieve single fundamental mode operation in VCSELs have been reported. The simplest approach is reducing the oxide [2] or implant [3] cavity diameter, but this comes at the expense of high current density and inferior laser reliability. Other approaches include a hybrid combination of oxide/implant apertures [4], surface 


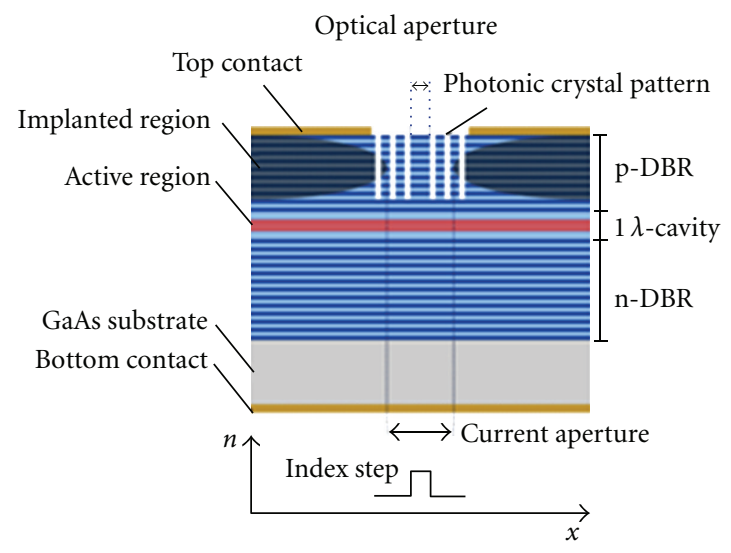

(a)

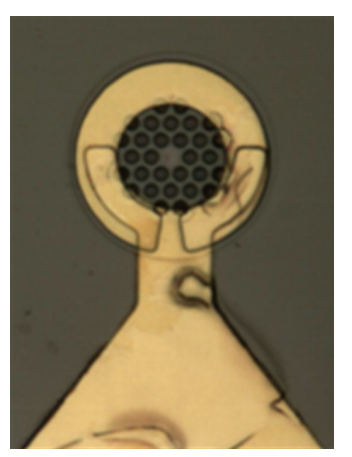

(b)

FIGURE 1: (a) Cross-section sketch and (b) top view image of planar ion implanted photonic crystal VCSEL.

relief etched structures [5], increasing the optical cavity length [6], etched holey patterns $[7,8]$, and etched photonic crystal VCSELs [9-14].

In most cases, the technique for achieving single mode operation requires an added epitaxial semiconductor layer or etched feature that has a stringent dimension control related to the wavelength. All of these approaches favor single fundamental mode operation by either increasing the gain for the fundamental mode, increasing the loss of higher order modes, or by engineering the transverse index profile of the VCSEL to support only the lowest order mode.

In this review, we will focus on index-guided single mode photonic crystal (PhC) VCSELs. In these devices, a 2dimensional periodic pattern of holes is etched into the top distributed Bragg reflector mirror (DBR). The lasing cavity is defined by the absence of one or more holes as depicted in Figure 1. Unlike in-plane 2-dimensional photonic crystal structures where the defect mode often lies within a frequency bandgap, the type of out-of-plane defect mode in the PhC VCSEL is not created by a photonic band gap. Instead, the PhC VCSEL out-of-plane mode is confined similarly to the case in a solid-core photonic crystal fiber, which guides by way of total internal reflection [15]. As we will show, the unetched defect has a higher refractive index as compared to the surrounding etched photonic crystal region.

The refractive index and optical loss arising from the $\mathrm{PhC}$ region can be engineered via the photonic crystal parameters of hole arrangement, period, diameter, and etch depth. A key feature is the periodicity of the etched pattern of holes which allows us to accurately calculate the effective refractive index. The modified index is not a geometric average of the low index etched regions within the semiconductor, but is actually much less [12]. Note that nonperiodic but symmetric etched features, such as wedges or rings, can also be used to engineer the refractive index $[16,17]$. However, this approach does rely upon geometrically averaging the index and thus is very sensitive to feature size and fabrication error. Moreover, etched features that are continuous, such as rings, will lead to degraded electrical injection.
The index confinement and optical loss can be exploited to create fundamental mode photonic crystal VCSELs. As shown in the comparison of Figure 2, conventional multimode VCSELs can be altered to show single mode lasing emission more than $30 \mathrm{~dB}$ above the nonlasing modes. Moreover, insuring single mode behavior can be maintained independent of the lasing wavelength or crystal design [18], precluding the need for accurate and/or wavelength specific features. Finally, the electrical diameter can be increased independent to the optical cavity to enable lower current density operation $[13,19]$ and thus potentially long laser lifetime [20].

In Section 2, we review the fabrication details for both oxide-confined and ion implanted PhC VCSELs. In Section 3, a description of the design approach which incorporates both index and loss confinement effects from the photonic crystal is introduced. In Section 4, the performance of various PhC VCSELs are presented. Finally, Section 5 summaries this review.

\section{Fabrication}

The epitaxial materials used are conventional allsemiconductor VCSELs consisting of upper p-type and lower n-type DBR mirrors which surround a quantum well active region [1]. In our studies, we have fabricated $\mathrm{PhC}$ VCSELs grown on GaAs substrates which emit at 670, 780, 850,980 , or $1300 \mathrm{~nm}[18,21]$. The device fabrication for oxide-confinement or ion implantation follows the typical steps as detailed below, with the addition of the patterning and anisotropic etching of the photonic crystal. In our PhC VCSELs, the optical cavity confinement is determined by the photonic crystal parameters of hole diameter $(a)$, period (b), and hole depth, whereas the electrical confinement is provided by the proton-implanted or oxide aperture. This allows for precise engineering of the index guidance effect of the photonic crystal independent of the electrical confinement of the VCSEL.

Implant-confined photonic crystal VCSELs are fabricated as follows [1]. Ohmic ring contacts (Ti/Au) for the top 


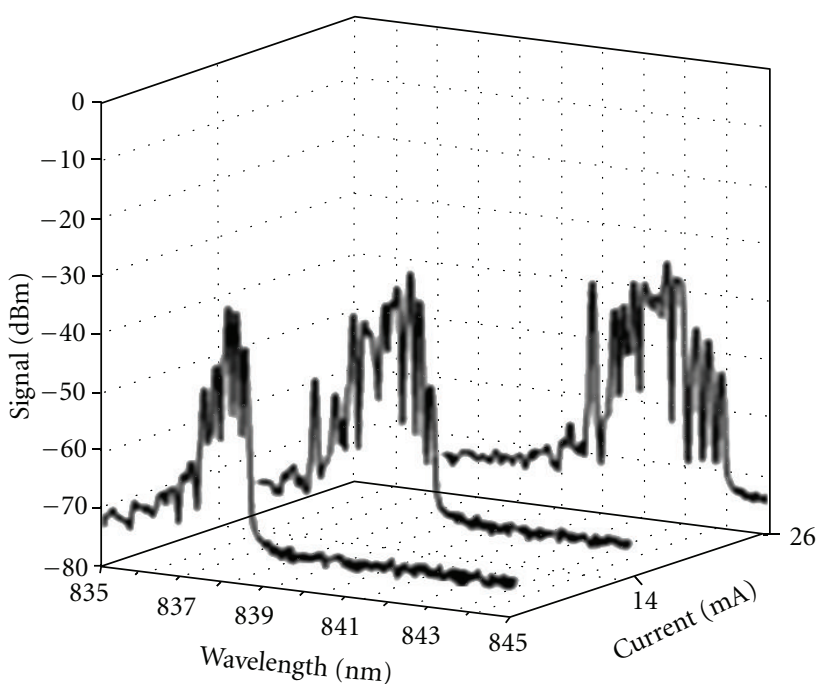

(a)

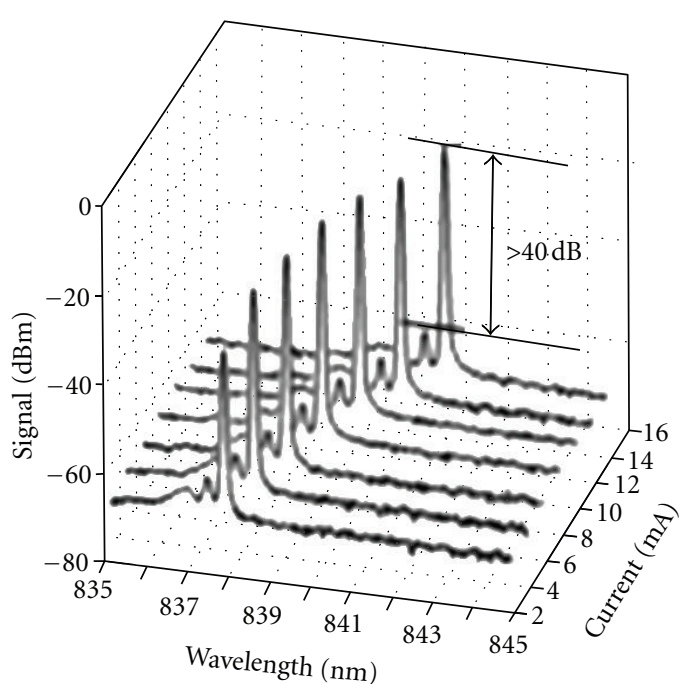

(b)

FIgURE 2: Optical spectrum of (a) oxide-confined and (b) photonic crystal VCSEL.

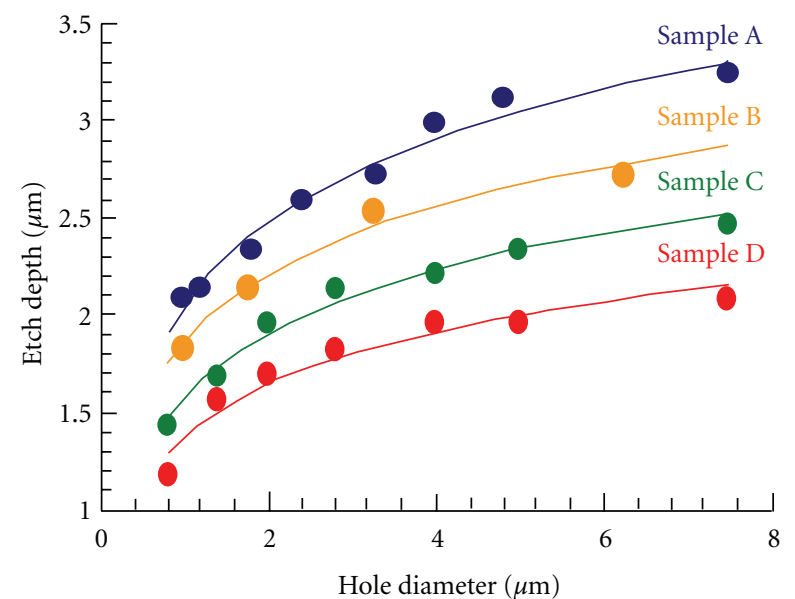

Figure 3: Etch depth versus hole diameter for $b / a=0.4,0.5,0.6$, and 0.7 .

p-type DBR are patterned using optical photolithography, deposited by electron-beam evaporation, and formed using conventional liftoff. A backside contact $(\mathrm{AuGe} / \mathrm{Ni} / \mathrm{Au})$ is deposited to form an ohmic contact to the n-type substrate. A thin protective layer of $\mathrm{SiO}_{2}$ is deposited, and thick $(12 \mu \mathrm{m})$ photoresist pillars are patterned in the center of each top ring contact with diameter varying from 6 to $20 \mu \mathrm{m}$. The exposed $\mathrm{SiO}_{2}$ is etched using $\mathrm{CF}_{4}$ reactive ion etching (RIE). These pillars serve to mask and define the apertures for the implant process. Protons are next implanted at typically $340 \mathrm{keV}$ (the implant energy will depend on the intended implant projected range [1]) with a dose of $4 \times 10^{14} \mathrm{~cm}^{-2}$ and afterwards the resist pattern is removed. Another larger resist mask is used for a second multiple implantation step to create a stacked implant from the implant aperture to the surface for electrical isolation between devices in a planar device topology. The implant isolation can be done either before or after the photonic crystal etch.

The photonic crystal patterns are defined by optical lithography in an $\mathrm{SiO}_{2}$ mask. Note that the periodic index variation of the hole pattern needs not be of the same size as the light wavelength, since the light is propagating parallel to the hole patterns [12]; hence micron-sized holes are sufficient, which allows for optical photolithographic patterning. The photonic crystal holes are etched into the top DBR using $\mathrm{SiCl}_{4} / \mathrm{Ar}$ inductively coupled plasma RIE. The etch rate of the holes will depend on their diameter, $b$, due to size-dependent etch effects [22]. The etch depth for the samples A, B, C, and D, which have a $b / a=0.4,0.5,0.6$, and 0.7 , respectively, is shown in Figure 3 as a function of photonic crystal hole diameter $(b)$. The holes were inspected in a scanning electron microscope at an angle of $35^{\circ}$ offnormal, and the etch depth was calculated by counting the number of DBR periods etched and multiplying by the thickness of one period. For a given etching time, smaller diameter holes etched to a shallower depth. The etch depth data can be empirically fit with a logarithmic function. The hole etch depth is limited by the large aspect ratio, but as we show below etching to and/or through the active region is undesirable. However, an important consequence is that the optical mode only experiences the photonic crystal in part of the longitudinal cavity; we will show below that this creates optical loss and confinement. After etching the photonic crystal pattern, the oxide mask is removed by $\mathrm{CF}_{4} \mathrm{RIE}$, and the samples are subjected to $30 \mathrm{sec}$ rapid thermal annealing at $325^{\circ} \mathrm{C}$ for ohmic contact formation. Figure 1 (a) illustrates a schematic of a completed implant-confined photonic crystal VCSEL and Figure 1(b) shows a top view of a planar implant PhC VCSEL.

For oxide-confined VCSELs, a high aluminum content layer $\left(\mathrm{Al}_{0.98} \mathrm{Ga}_{0.02} \mathrm{As}\right)$ is placed above the active region for 


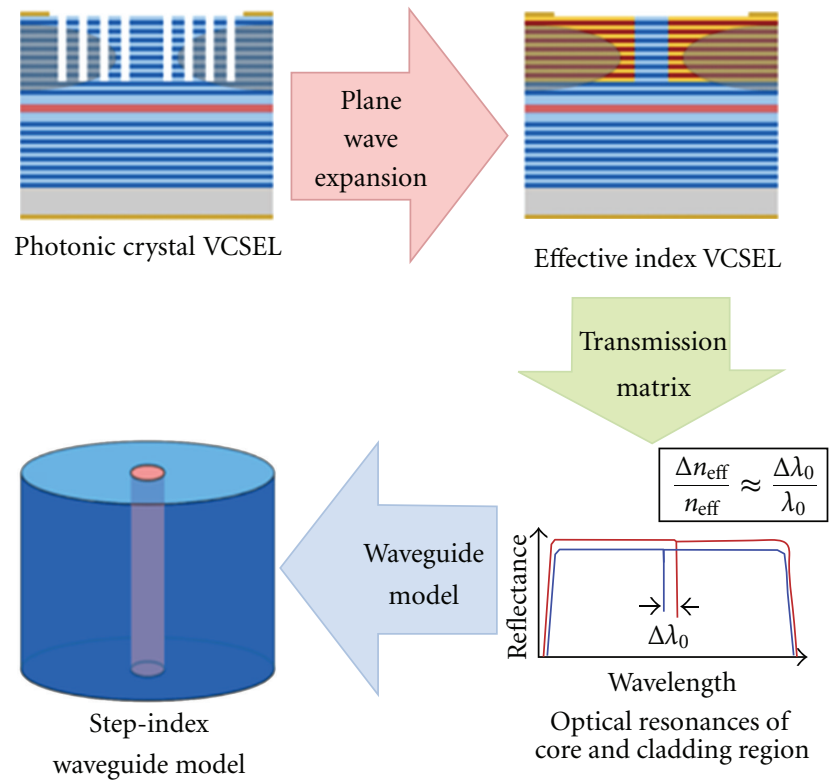

FIgURE 4: Design approach for photonic crystal VCSELs.

fabrication of an oxide aperture $[1,23]$. After top and bottom contact metals are defined and deposited, photonic crystal patterns and an oxidation mesa or trench are defined by standard optical lithography and are etched by ICP-RIE. The hole pattern and the oxidation trench are simultaneously patterned by optical lithography but can be etched separately or simultaneously [24]. This results in self-alignment of the $\mathrm{PhC}$ defect to the oxide aperture. After removal of the etch mask, selective oxidation at $420^{\circ} \mathrm{C}$ in a steam environment creates the oxide aperture.

The index step of the PhC defect cavity can be engineered by carefully designing the periodic hole pattern as well as by controlling the etch depth of the air holes. However, to simply achieve single fundamental mode operation, $\mathrm{PhC}$ parameters such as lattice constant, hole diameter, or etching depths of the air holes do not need to be stringently controlled [18]. The large tolerances in the fabrication process makes PhC VCSELs suitable for mass-production and thus an excellent candidate for low cost/high volume consumer applications.

\section{Design}

The design of the photonic crystal has been developed to incorporate both the index confinement [10] as well as the confinement that arises due to optical loss [25, 26]. The photonic crystal has a reduced effective refractive index, due to the periodic refractive index variation (see Figure 1). The basic design approach is schematically shown in Figure 4. The VCSEL is modeled using a simplified stepindex optical fiber waveguide model: the defect in the photonic crystal is considered to be the core of the fiber with index $n_{\text {core }}$, and the photonic crystal region surrounding is taken as the reduced-index cladding region with $n_{\text {clad }}$. More computationally intensive and rigorous methods, such

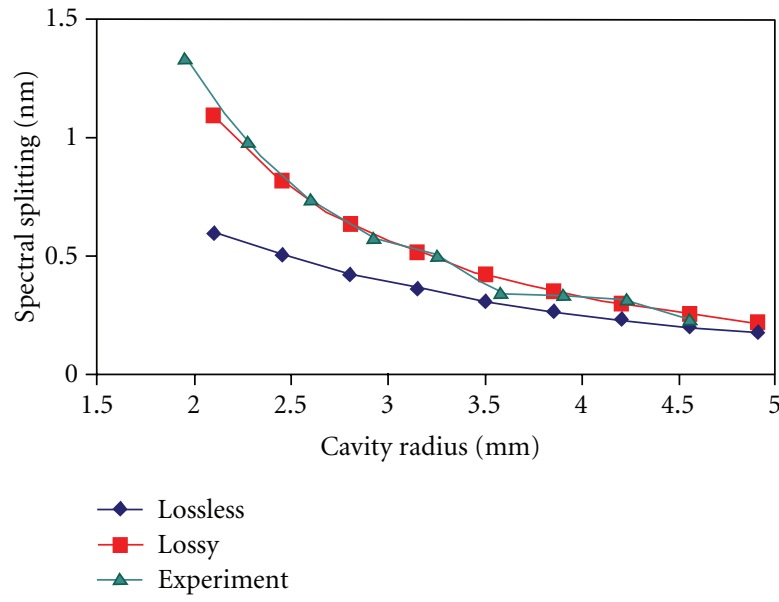

Figure 5: Spectral splitting between fundamental and first higher order mode versus cavity radius.

as finite-difference time-domain, finite element, or vectorial and three-dimensional calculations [27-29] can also be employed.

The waveguiding and optical loss effects are incorporated by using a complex refractive index in the $\mathrm{PhC}$ clad region. The real part of the effective refractive index of the $\mathrm{PhC}$ cladding is found from the band diagram calculated using the plane wave expansion method. The slope of the band for out-of-plane propagation determines the effective refractive index for each high and low index layer of the DBR mirror. This effective homogeneous index is used to replace the DBR layers that are penetrated by the photonic crystal, thereby accounting for the finite etch depth. The index step difference between the core and clad regions can be found using the difference in the calculated resonance wavelengths [30] of a matrix calculation [26]. This procedure intrinsically accounts for the finite etch depth of the holes.

The optical loss introduced by the finite etch depth of the holes is accounted for using a complex refractive index in the cladding region [31]:

$$
n_{\text {clad }}=n_{\text {PhC }}^{\prime}+\mathrm{i} n_{\text {loss }}^{\prime \prime}
$$

To determine the magnitude of the imaginary component, we rely upon the confinement induced by the loss. This can be inferred from the cold cavity spectral splitting between the modes observed below threshold, as discussed below. From the Helmholtz equation:

$$
\nabla^{2} U+n^{2}(r) k_{0}^{2} U=0
$$

where $U$ is the field in three spatial dimensions, $n$ is the radial-dependent refractive index profile, and $k_{0}$ is the freespace wave number, we assume separable solutions of the form:

$$
U(r, \phi, z)=u(r) e^{-i m \phi} e^{-i k_{z} z},
$$

where $u$ is the radial field profile, $m$ is an integer, and $k_{z}$ is an effective propagation constant. The propagation constant $k_{z}$ 


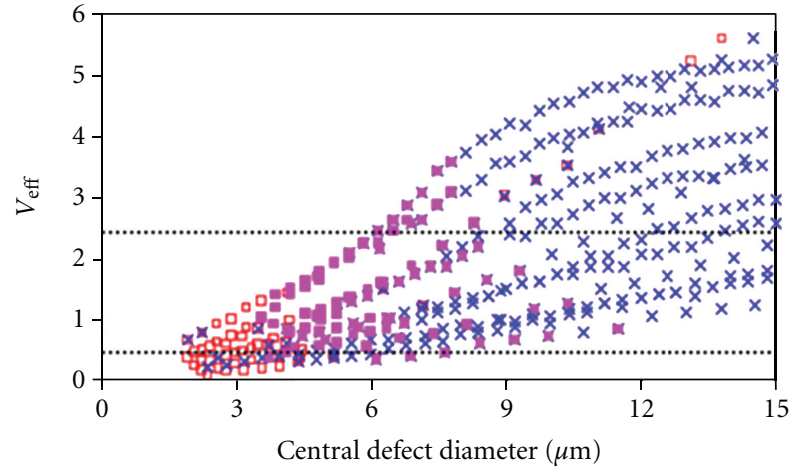

- Not lasing

$\times$ Multimode

- Single mode

FigURE 6: Normalized frequency and modal properties of oxideconfined $850 \mathrm{~nm}$ VCSELs.

is set by Fabry-Perot cavity. Inserting the solutions in (3) into (2) gives

$$
\frac{d^{2} u}{d r^{2}}+\frac{1}{r} \frac{d u}{d r}+\left(n^{2}(r) k_{0}^{2}-k_{z}^{2}-\frac{m^{2}}{r^{2}}\right) u(r)=0
$$

Solving the eigenvalue equation (4) using finite differences produces eigenvectors $u$ and eigenvalues $k_{0}$, which are set of solutions for the resonant modes of the waveguide whose wave numbers are $k_{0}$. The resonant wavelength is

$$
\lambda_{0}=\frac{2 \pi c}{\operatorname{Re}\left\{\omega_{0}\right\}}=\frac{2 \pi}{\operatorname{Re}\left\{k_{0}\right\}},
$$

and the loss experienced by the mode is

$$
\alpha_{\mathrm{i}}=\operatorname{Im}\left\{k_{0}\right\} .
$$

We compare the calculated resonances (fundamental and first higher order mode) to spectral measurements from a fabricated PhC VCSEL with injection current less than threshold [25]. Figure 5 shows spectral splitting between the fundamental and first higher order mode as a function of cavity radius. The lossless (lossy) curve corresponds to a real (complex) PhC refractive index used to solve (4). Note that the splitting increases for reduced cavity size but is greater for a nonzero $n_{\text {loss }}^{\prime}$. By varying this parameter, we can match the experimental curve shown in Figure 5. Hence, the effect of optical loss from the photonic crystal is to increase the cavity confinement.

For a lossless waveguide, the modal properties can be quantified from the normalized frequency parameter, $V_{\text {eff: }}$

$$
V_{\text {eff }}=\frac{2 \pi R}{\lambda} \sqrt{n_{\text {core }}^{2}-n_{\text {clad }}^{2}},
$$

where $R=a-b / 2$ is the core radius and $\lambda$ is the freespace optical wavelength. For a lossless guide, the singlemode cutoff is $V_{\text {eff }}<2.405$ [32]. Note that in (7), the finite etch depth dependence is accounted in the determination of the purely real $n_{\text {clad. }}$ In the next section, we compare the

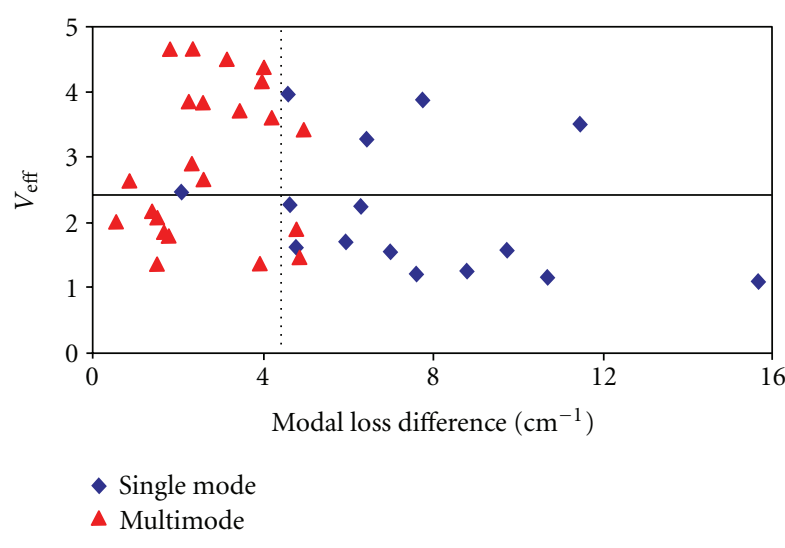

FIGURE 7: Normalized frequency versus modal loss difference between fundamental and first higher order mode of oxideconfined $850 \mathrm{~nm}$ VCSELs.

calculated values of $V_{\text {eff }}$ and the extracted values of optical loss, with the modal characteristics of PhC VCSELs.

\section{Performance}

The characteristics of PhC VCSELs were measured using onwafer probing at room temperature. For continuous wave light versus current measurements, the input current was varied and both the device voltage and the output from a silicon photodetector were measured using a semiconductor parameter analyzer. The spectral characteristics were measured using an optical spectrum analyzer with resolution bandwidth of $0.06 \mathrm{~nm}$. At maximum power, each VCSEL was categorized as either not lasing, multimode lasing, or single mode lasing, where single mode is defined as a single spectral peak at the fundamental (lowest energy) wavelength with greater than $30 \mathrm{~dB}$ side mode suppression ratio. Note that VCSELs that lased single mode at threshold with higher order mode lasing at maximum power are defined as multimode.

In Figure 6 we show the calculated $V_{\text {eff }}$ and the observed modal properties for approximately 1500 different photonic crystal designs with differing period, b/a ratios, and etch depth [33]. For many of these $850 \mathrm{~nm}$ oxide-confined VCSELs with $V_{\text {eff }}<2.4$, single mode lasing is observed, particularly for cavity diameters less than $6 \mu \mathrm{m}$. However, multi-mode VCSELs with $V_{\text {eff }}<2.4$ can be seen, as well as single mode PhC VCSELs with $V_{\text {eff }}>2.4$. Hence, the loss induced confinement is a critical design parameter.

In Figure 7, we show a smaller population of approximately 40 oxide-confined $850 \mathrm{~nm}$ photonic crystal VCSELs with differing designs where $V_{\text {eff }}$ and the modal loss difference between the fundamental and the first higher order mode are determined. The single mode cutoff is shown by a horizontal line in Figure 7. Again, we observe that the normalized frequency parameter is not a good determination for single mode VCSELs. However, if optical loss is considered, a cutoff can be seen between single and multimode lasing at approximately $5 \mathrm{~cm}^{-1}$ of loss difference 


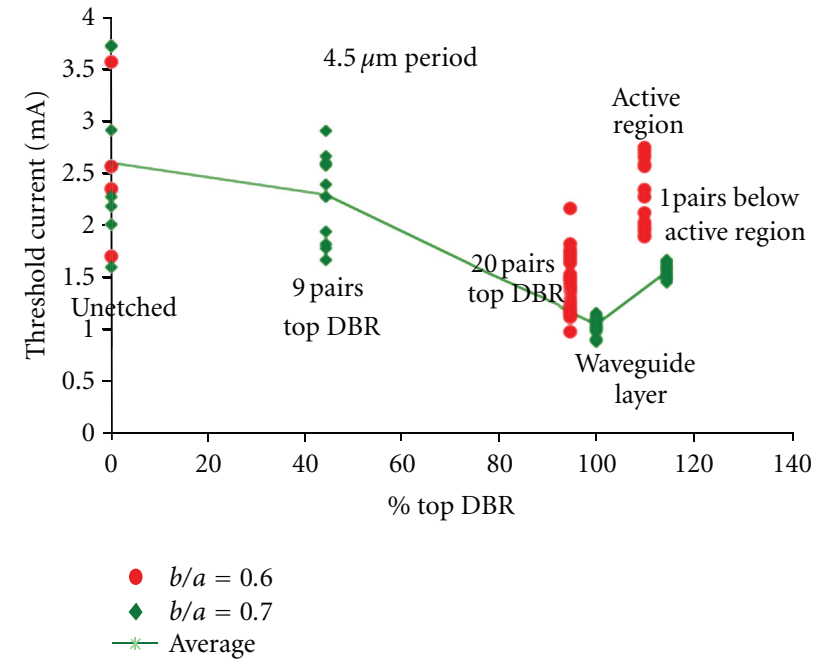

(a)

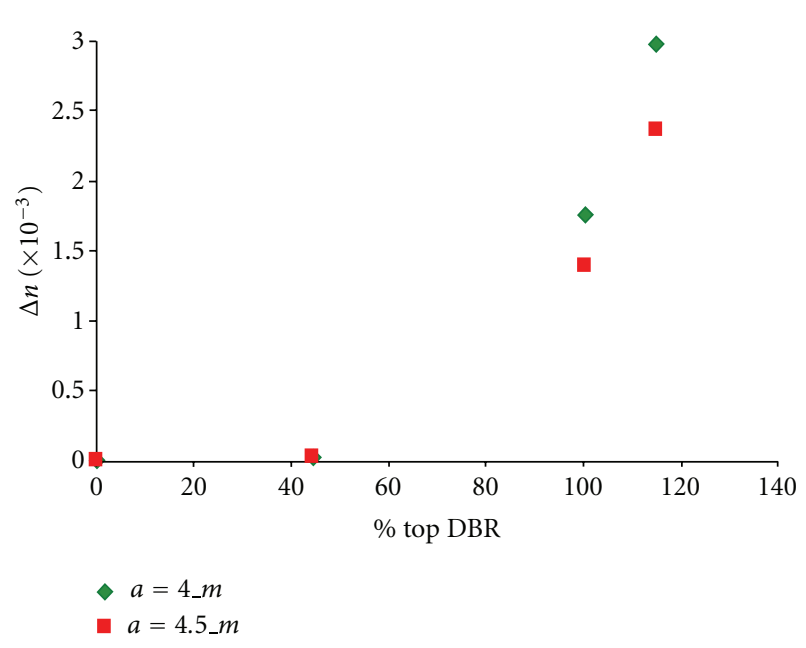

(b)

FIGURE 8: (a) Threshold current and (b) index contrast versus etch depth into top DBR for ion implanted 850 nm VCSELs.

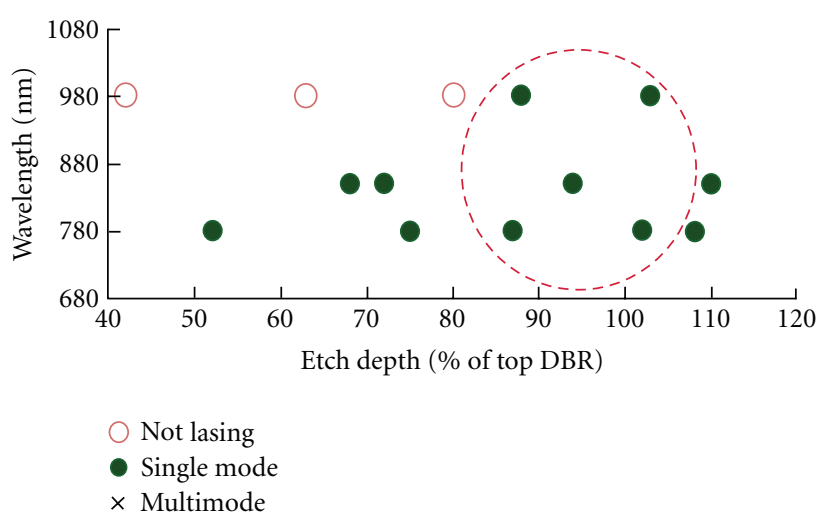

(a)

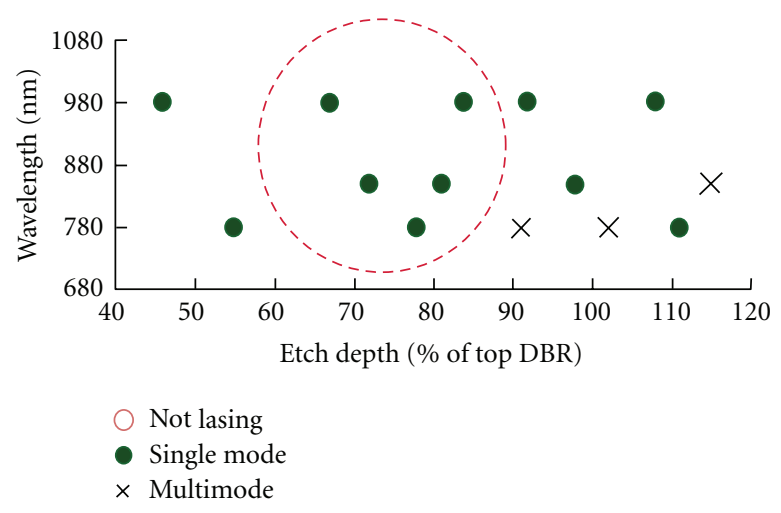

(b)

Figure 9: Modal properties of oxide-confined PhC VCSELs; designs that are independent of wavelength are shown in dashed circles: (a) $b / a$ $=0.7, \mathrm{a}=4 \mu \mathrm{m}$, oxide aperture $=12 \mu \mathrm{m}$ and (b) $b / a=0.7, \mathrm{a}=4.5 \mu \mathrm{m}$, oxide aperture $=12 \mu \mathrm{m}[18]$.

(dotted vertical line in Figure 7). This condition corresponds to the point at which the loss to higher order mode is too great for gain to compensate and thus lasing to occur.

The effect of the etch depth on confinement is explicitly shown for implanted $850 \mathrm{~nm}$ photonic crystal VCSELs in Figure 8(a). Here, we calculate the difference between the core and clad index as a function of the percent etch depth into the top DBR for two b/a ratios. The confinement increases as the hole depth increases. However, etching through the active region leads to increased threshold current, as seen in Figure 8(b). Multiple VCSELs with two b/a ratios etched at different depths into the top DBR are shown. The highest threshold currents are for lasers with no etching, which arises from the diffraction loss inherent to ion implanted VCSELs [34]. The threshold current decreases with increased etch depth (increased confinement), reaching the lowest values when the photonic crystal is etched precisely through the top DBR. However, when the VCSELs are etched through the active region, the threshold current increases, presumably due to nonradiative recombination occurring at the edges of the penetrating holes into the quantum wells.

A notable feature of photonic crystal designs is that the designs are wavelength agnostic. In Figure 9, we present the modal properties of 780, 850, and $980 \mathrm{~nm}$ VCSELs, all with the same photonic crystal pattern [18]. In this study, single mode operation is defined as $>35 \mathrm{~dB}$ side mode suppression, and all lasers emit $>1 \mathrm{~mW}$ of single mode output. In other studies, we have achieved as high as $3 \mathrm{~mW}$ of fundamental mode emission [13].

For Gaussian fundamental mode emission, we expect to see a Gaussian far field mode with a minimum of divergence. The far field divergence for visible implanted PhC VCSELs is plotted in Figure 10 as a function of their measured side mode suppression ratio (SMSR). The insets in Figure 10 are false color representation of the far field intensity 


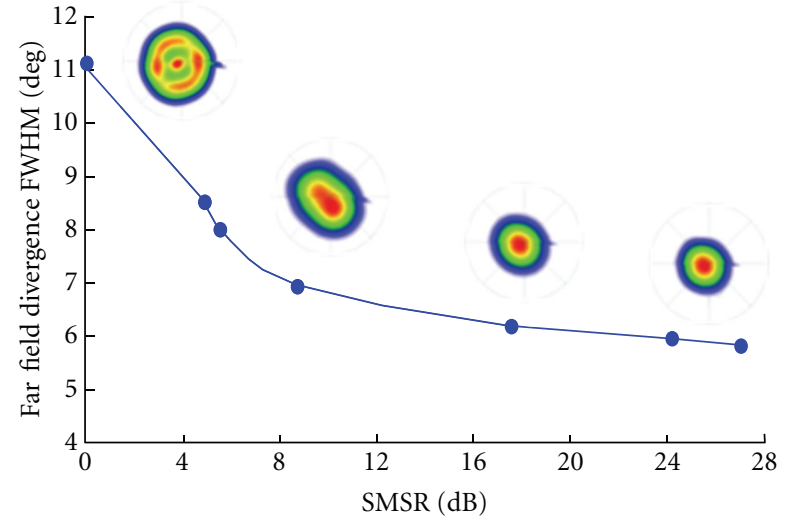

FIgURe 10: Far field divergence of implanted $670 \mathrm{~nm}$ PhC VCSELs as a function of side mode suppression ratio; the insets show representative far field patterns.

patterns. For multimode VCSELs (0 dB SMSR), a ring-like far field is observed. For increasing single mode operation, the divergence angle monotonically decreases.

\section{Summary}

Implant and oxide-confined photonic crystal VCSELs emitting from 670 to $1300 \mathrm{~nm}$ were designed, fabricated, and characterized to parametrically study the effects of photonic crystal design and etch depth on single mode operation. A lossy model for the photonic crystal waveguide used for transverse confinement in VCSELs has been developed. We have found a wide range of designs and fabrication approaches to achieve reproducible single fundamental mode operation. Moreover, these designs are found to be independent of wavelength and lead to $1-3 \mathrm{~mW}$ of output power in single aperture devices.

These developments in the control of transverse modes can significantly advance the use of VCSELs for a number of applications. With the methods developed here, photonic crystal VCSELs can be designed to operate only on a single mode while maintaining a larger aperture size and higher efficiency. Thus, these advancements could enable a single mode VCSEL with high output powers and greater reliability for sensing and long-haul communication applications.

\section{Acknowledgments}

The authors thank many former University of Illinois students for extensive VCSEL characterization. This work was partially supported by the National Science Foundation under Awards nos. 03-35082, DMI 0328162, and NDSEG Graduate Fellowship.

\section{References}

[1] K. D. Choquette and K. M. Geib, "Fabrication and performance of vertical cavity surface emitting lasers," in Vertical Cavity Surface Emitting Lasers, C. Wilmsen, H. Temkin, L. Coldren et al., Eds., chapter 5, Cambridge University Press, Cambridge, UK, 1999.
[2] C. Jung, R. Jager, M. Grabherr et al., " $4.8 \mathrm{~mW}$ single-mode oxide confined top surface emitting vertical-cavity laser diodes," Electronics Letters, vol. 33, no. 21, pp. 1790-1791, 1997.

[3] R. A. Morgan, G. D. Guth, M. W. Focht et al., "Transverse mode control of vertical-cavity top-surface-emitting lasers," IEEE Photonics Technology Letters, vol. 5, no. 4, pp. 374-377, 1993.

[4] E. W. Young, K. D. Choquette, S. L. Chuang, K. M. Geib, A. J. Fischer, and A. A. Allerman, "Single-transverse-mode verticalcavity lasers under continuous and pulsed operation," IEEE Photonics Technology Letters, vol. 13, no. 9, pp. 927-929, 2001.

[5] H. Martinsson, J. A. Vukušić, M. Grabherr et al., "Transverse mode selection in large-area oxide-confined vertical-cavity surface-emitting lasers using a shallow surface relief," IEEE Photonics Technology Letters, vol. 11, no. 12, pp. 1536-1538, 1999.

[6] H. J. Unold, S. W. Z. Mahmoud, R. Jager, M. Kicherer, M. C. Riedl, and K. J. Ebeling, "Improving single-mode VCSEL performance by introducing a long monolithic cavity," IEEE Photonics Technology Letters, vol. 12, no. 8, pp. 939-941, 2000.

[7] A. Furukawa, S. Sasaki, M. Hoshi, A. Matsuzono, K. Moritoh, and T. Baba, "High-power single-mode vertical-cavity surfaceemitting lasers with triangular holey structure," Applied Physics Letters, vol. 85, no. 22, pp. 5161-5163, 2004.

[8] P. O. Leisher, A. J. Danner, J. J. Raftery Jr., and K. D. Choquette, "Proton implanted singlemode holey vertical-cavity surfaceemitting lasers," Electronics Letters, vol. 41, no. 18, pp. 10101011, 2005.

[9] D. S. Song, S. H. Kim, H. G. Park, C. K. Kim, and Y. H. Lee, "Single-fundamental-mode photonic-crystal vertical-cavity surface-emitting lasers," Applied Physics Letters, vol. 80, no. 21, p. 3901, 2002.

[10] N. Yokouchi, A. J. Danner, and K. D. Choquette, "Two-dimensional photonic crystal confined vertical-cavity surface-emitting lasers," IEEE Journal on Selected Topics in Quantum Electronics, vol. 9, no. 5, pp. 1439-1445, 2003.

[11] A. J. Danner, J. J. Raftery Jr., T. Kim, P. O. Leisher, A. V. Giannopoulos, and K. D. Choquette, "Progress in photonic crystal vertical cavity lasers," IEICE Transactions on Electronics, vol. E88-C, no. 5, pp. 944-949, 2005.

[12] N. Yokouchi, A. J. Danner, and K. D. Choquette, "Etching depth dependence of the effective refractive index in two-dimensional photonic-crystal-patterned vertical-cavity surface-emitting laser structures," Applied Physics Letters, vol. 82, no. 9, pp. 1344-1346, 2003.

[13] A. J. Danner, T. S. Kim, and K. D. Choquette, "Single fundamental mode photonic crystal vertical cavity laser with improved output power," Electronics Letters, vol. 41, no. 6, pp. 325-326, 2005.

[14] H. P. D. Yang, F. I. Lai, Y. H. Chang et al., "Singlemode $(\mathrm{SMSR}<40 \mathrm{~dB})$ proton-implanted phptonic crystal verticalcavity surface-emitting lasers," Electronics Letters, vol. 41, no. 6, pp. 326-328, 2005.

[15] T. A. Birks, J. C. Knight, and P. S. J. Russell, "Endlessly singlemode photonic crystal fiber," Optics Letters, vol. 22, no. 13, pp. 961-963, 1997.

[16] A. Furukawa, S. Sasaki, M. Hoshi, A. Matsuzono, K. Moritoh, and T. Baba, "High-power single-mode vertical-cavity surfaceemitting lasers with triangular holey structure," Applied Physics Letters, vol. 85, no. 22, pp. 5161-5163, 2004. 
[17] P. O. Leisher, A. J. Danner, J. J. Raftery, D. Siriani, and K. D. Choquette, "Loss and index guiding in single-mode protonimplanted holey vertical-cavity surface-emitting lasers," IEEE Journal of Quantum Electronics, vol. 42, no. 10, pp. 1091-1096, 2006.

[18] A. M. Kasten, M. P. Tan, J. D. Sulkin, P. O. Leisher, and K. D. Choquette, "Photonic crystal vertical cavity lasers with wavelength-independent single-mode behavior," IEEE Photonics Technology Letters, vol. 20, no. 23, pp. 2010-2012, 2008.

[19] C. Chen, Z. Tian, K. D. Choquette, and D. V. Plant, "25-Gb/s direct modulation of implant confined holey vertical-cavity surface-emitting lasers," IEEE Photonics Technology Letters, vol. 22, no. 7, pp. 465-467, 2010.

[20] A. M. Kasten, J. D. Sulkin, P. O. Leisher, D. K. McElfresh, D. Vacar, and K. D. Choquette, "Manufacturable photonic crystal single-mode and fluidic vertical-cavity surface-emitting lasers," IEEE Journal on Selected Topics in Quantum Electronics, vol. 14, no. 4, pp. 1123-1131, 2008.

[21] P. O. Leisher, A. J. Danner, and K. D. Choquette, "Single-mode 1.3- $\mu \mathrm{m}$ photonic crystal vertical-cavity surface-emitting laser," IEEE Photonics Technology Letters, vol. 18, no. 20, pp. 21562158, 2006.

[22] R. A. Gottscho and C. W. Jurgensen, "Microscopic uniformity in plasma etching," Journal of Vacuum Science \& Technology B, vol. 10, p. 2133, 1992.

[23] K. D. Choquette, R. P. Schneider Jr., K. L. Lear, and K. M. Geib, "Low threshold voltage vertical-cavity lasers fabricated by selective oxidation," Electronics Letters, vol. 30, no. 24, pp. 2043-2044, 1994.

[24] M. S. Alias, S. Shaari, P. O. Leisher, and K. D. Choquette, "Single transverse mode control of VCSEL by photonic crystal and trench patterning," Photonics and Nanostructures, vol. 8, no. 1, pp. 38-46, 2010.

[25] D. F. Siriani, P. O. Leisher, and K. D. Choquette, "Lossinduced confinement in photonic crystal vertical-cavity surface-emitting lasers," IEEE Journal of Quantum Electronics, vol. 45, no. 7, pp. 762-768, 2009.

[26] D. F. Siriani, M. P. Tan, A. M. Kasten et al., "Mode control in photonic crystal vertical-cavity surface-emitting lasers and coherent arrays," IEEE Journal on Selected Topics in Quantum Electronics, vol. 15, no. 3, Article ID 4781555, pp. 909-917, 2009.

[27] P. Bienstman, R. Baets, J. Vukusic et al., "Comparison of optical VCSEL models on the simulation of oxide-confined devices," IEEE Journal of Quantum Electronics, vol. 37, no. 12, pp. 1618-1631, 2001.

[28] T. Czyszanowski, M. Dems, and K. Panajotov, "Single mode condition and modes discrimination in photonic-crystal $1.3 \mu \mathrm{m}$ AlInGaAs/InP VCSEL," Optics Express, vol. 15, no. 9, pp. 5604-5609, 2007.

[29] T. Czyszanowski, M. Dems, and K. Panajotov, "Optimal parameters of photonic-crystal vertical-cavity surfaceemitting diode lasers," Journal of Lightwave Technology, vol. 25, no. 9, pp. 2331-2336, 2007.

[30] G. R. Hadley, "Effective index model for vertical-cavity surface-emitting lasers," Optics Letters, vol. 20, no. 13, pp. 1483-1485, 1995.

[31] A. E. Siegman, "Propagating modes in gain-guided optical fibers," Journal of the Optical Society of America A, vol. 20, no. 8, pp. 1617-1628, 2003.
[32] R. S. Quimby, Photonics and Lasers, John Wiley \& Sons, New Jersey, NJ, USA, 2006.

[33] A. J. Danner, J. J. Raftery Jr., P. O. Leisher, and K. D. Choquette, "Single mode photonic crystal vertical cavity lasers," Applied Physics Letters, vol. 88, no. 9, Article ID 091114, 2006.

[34] G. Hasnain, K. Tai, L. Yang et al., "Performance of gain-guided surface emitting lasers with semiconductor distributed Bragg reflectors," IEEE Journal of Quantum Electronics, vol. 27, no. 6, pp. 1377-1385, 1991. 

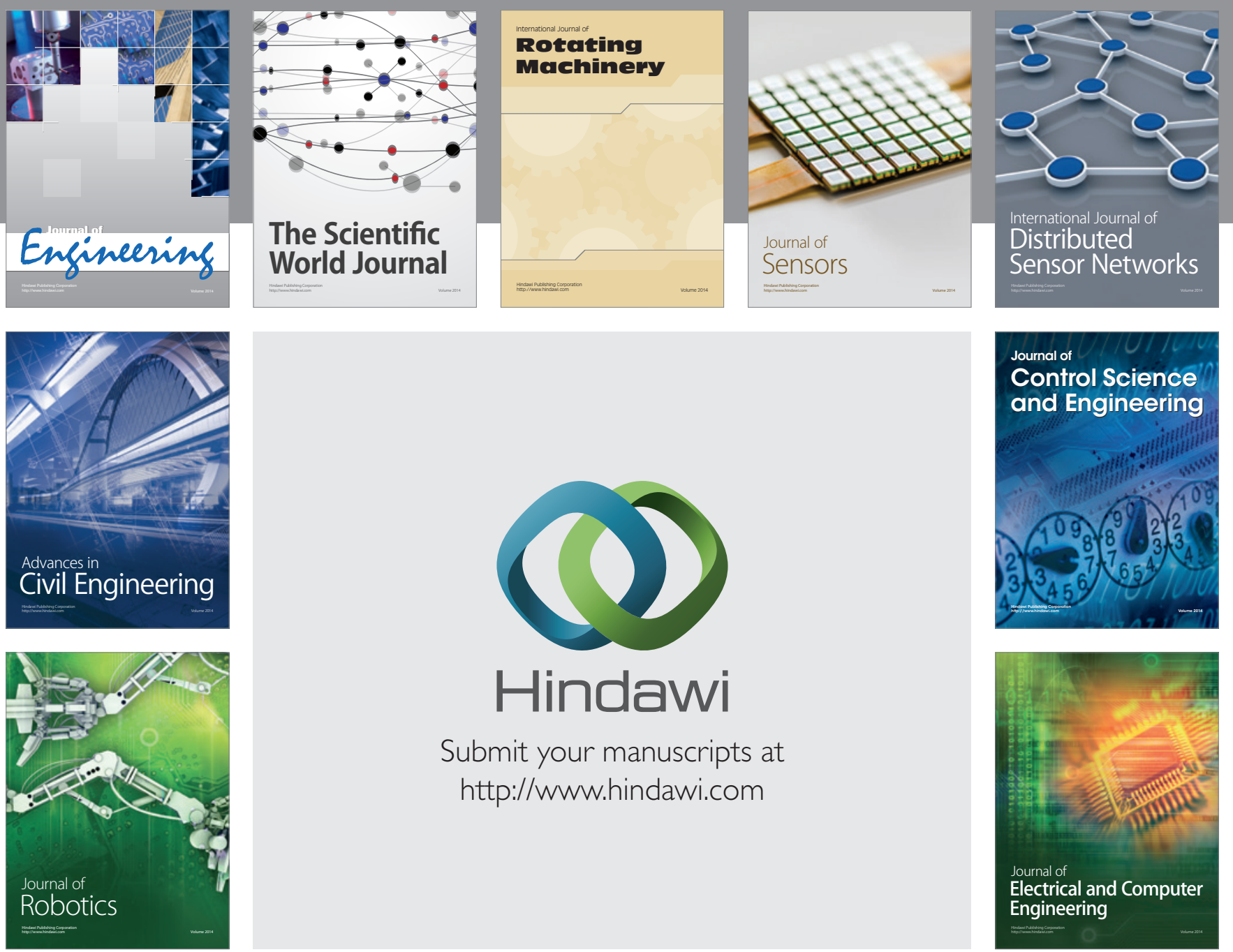

Submit your manuscripts at

http://www.hindawi.com
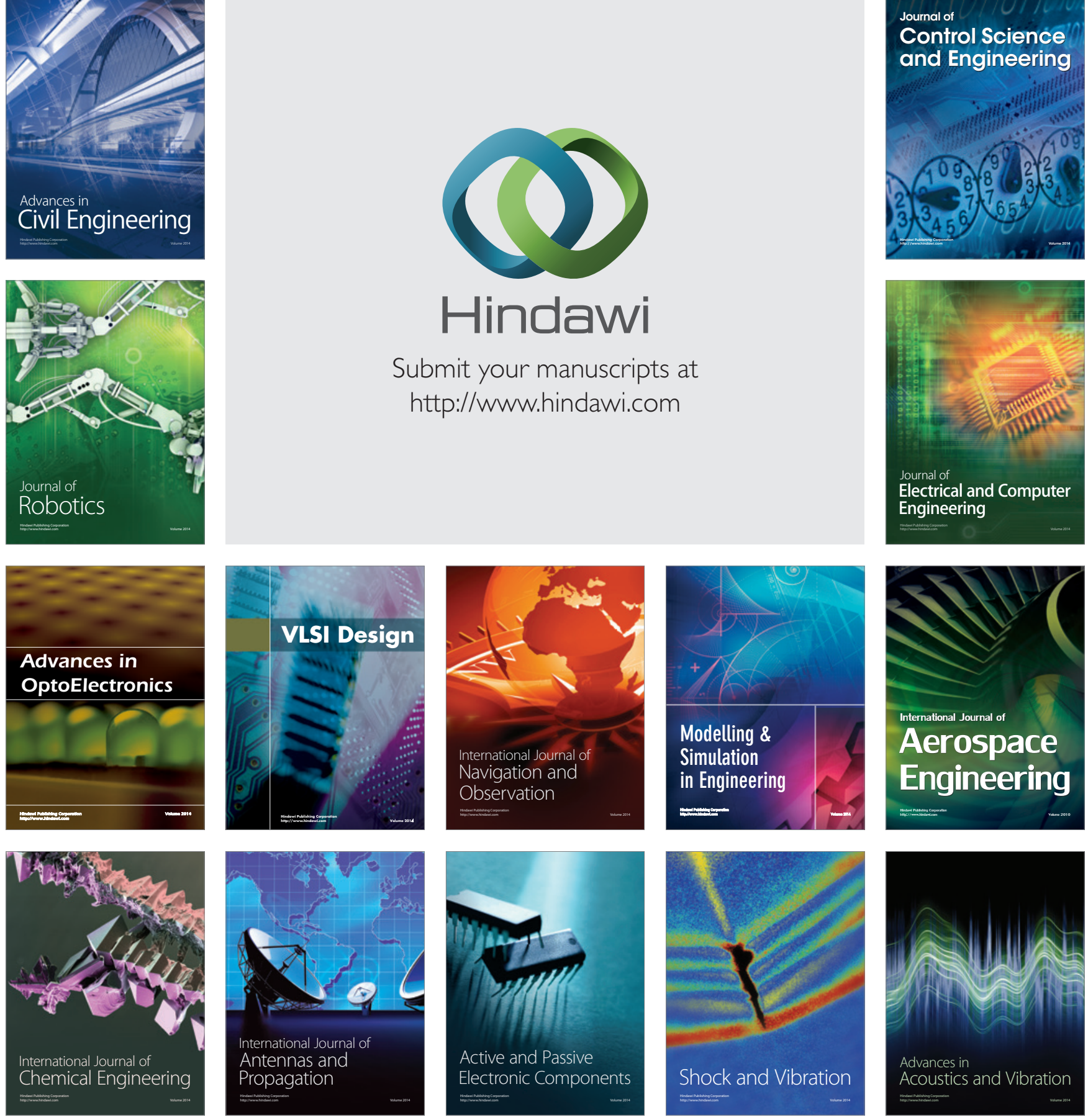